Draft version August 29, 2018

Preprint typeset using LATEX style emulateapj v. 12/16/11

\title{
COMPACT BINARY MERGERS AND THE EVENT RATE OF FAST RADIO BURSTS
}

\author{
XiaO-Feng CaO ${ }^{1}$, Yun-Wei $\mathrm{Yu}^{2,3}$, ANd Xia Zhou ${ }^{4}$ \\ Draft version August 29, 2018
}

\begin{abstract}
Fast radio bursts (FRBs) are usually suggested to be associated with mergers of compact binaries consisting of white dwarfs (WDs), neutron stars (NSs), or black holes (BHs). We test these models by fitting the observational distributions in both redshift and isotropic energy of 22 Parkes FRBs, where, as usual, the rates of compact binary mergers (CBMs) are connected with cosmic star formation rates by a power-law distributed time delay. It is found that the observational distributions can well be produced by the CBM model with a characteristic delay time from several ten to several hundred Myr and an energy function index $1.2 \lesssim \gamma \lesssim 1.7$, where a tentative fixed spectral index $\beta=0.8$ is adopted for all FRBs. Correspondingly, the local event rate of FRBs is constrained to $(3-6) \times 10^{4} f_{\mathrm{b}}^{-1}(\mathcal{T} / 270 \mathrm{~s})^{-1}(\mathcal{A} / 2 \pi)^{-1} \mathrm{Gpc}^{-3} \mathrm{yr}^{-1}$ for an adopted minimum FRB energy of $E_{\min }=$ $3 \times 10^{39} \mathrm{erg}$, where $f_{\mathrm{b}}$ is the beaming factor of the radiation, $\mathcal{T}$ is the duration of each pointing observation, and $\mathcal{A}$ is the sky area of the survey. This event rate, about an order of magnitude higher than the rates of NS-NS/NS-BH mergers, indicates that the most promising origin of FRBs in the CBM scenario could be mergers of WD-WD binaries. Here a massive WD could be produced since no FRB was found to be associated with a type Ia supernova. Alternatively, if actually all FRBs can repeat on a timescale much longer than the period of current observations, then they could also originate from a young active NS that forms from relatively rare NS-NS mergers and accretion-induced collapses of WD-WD binaries.
\end{abstract}

Subject headings: radio continuum: general — stars: neutron — stars: white dwarfs

\section{INTRODUCTION}

Studies on mergers of binary systems composing of a pair of compact objects, i.e., white dwarfs (WDs), neutron stars (NSs), or black holes (BHs), are of fundamental importance in astrophysics, because these mergers have or might have tight connections with current and future detections of gravitational waves Abbott et al. 2016, 2017), with the formation of heavy elements via $\mathrm{r}$ process (Lattimer \& Schramm 1974; Eichler et al. 1989; Hotokezaka et al. 2013; Bauswein et al. 2013; Just et al. 2015), with the production of type Ia supernovae ( $\mathrm{SNe}$ Ia; Tutukov \& Yungelson 1981; Webbink 1984), and with the origin of short gamma-ray bursts (GRBs: Paczynski 1986; Eichler et al. 1989; Guetta \& Piran 2006; Coward et al.|2012) as well as mergernova/kilonova emission (Li \& Paczyński 1998; Metzger et al. 2010; Yu et al. 2013; Coulter et al. 2017; Evans et al. 2017). The detection of various possible electromagnetic radiation from compact binary mergers $(\mathrm{CBMs})$ can play a crucial role in uncovering the nature of progenitor binaries and in locating and identifying the associated gravitational wave signals.

Recently, it was suggested that some CBMs, specifically, mergers of double WDs (Kashivama et al. 2013), of double NSs (Totani 2013; Wang et al. 2016; Yamasaki et al. 2017), of a NS and a BH

\footnotetext{
${ }^{1}$ School of Physics and Electronics Information, Hubei University of Education, Wuhan 430205, China

${ }^{2}$ Institute of Astrophysics, Central China Normal University, Wuhan 430079, China, yuyw@mail.ccnu.edu.cn

${ }^{3}$ Key Laboratory of Quark and Lepton Physics (Central China Normal University), Ministry of Education, Wuhan 430079, China

${ }^{4}$ Xinjiang Astronomical Observatory, Chinese Academy of Sciences, Urumqi 830011, China
}

(Mingarelli et al. 2015), or even of two charged BHs (Zhang 2016), could be responsible for the newly-discovered fast radio bursts (FRBs). FRBs are millisecond radio transients of intensities of a few to a few tens of Jansky at $\sim 1$ $\mathrm{GHz}$ (Lorimer et al. 2007; Keane et al. 2012, 2016; Thornton et al. 2013; Burke-Spolaor \& Bannister 2014; Spitler et al. 2014; Ravi et al.] 2015; Masui et al. 2015; Champion et al. 2016; Caleb et al. 2017; Petroff et al. 2017; Bannister et al. 2017). Due to the short durations of FRBs and the low angular resolution of present radio surveys, it is difficult to capture counterparts of FRBs in other wavelength bands, even if these couterparts indeed exist. This makes it impossible to directly determine the distances of FRB\&5 and to identify their origins. In any case, the anomalously high dispersion measures (DMs; $\sim 200-2600 \mathrm{pc} \mathrm{cm}^{-3}$ ) of FRBs, which are too high to be accounted for by the high-latitude inter-stellar medium in the Milky Way, robustly suggest that the FRBs could have cosmological distances of redshifts up to $z \sim 4.0$. Therefore, the isotropically equivalent energy release of an FRB can be estimated to within the range of $\sim 10^{39-42} \mathrm{erg}$. In the suggested CBM models, such an energy could be naturally provided by the inspiral of the binary or the spin-down of the remnant object due to magnetic dipole radiation and magnetospheric activities. Furthermore, it is believed that this energy should be released via coherent radiations, with some similarity to the pulse radiation of pulsars (Yang \& Zhang 2017a).

\footnotetext{
${ }^{5}$ For the only repeated FRB, FRB 121102 , its host galaxy and a persistent radio counterpart have been detected and then its redshift has been measured to $z=0.19$, which undeniably confirmed its cosmological origin (Chatteriee et al. 2017; Marcote et al. 2017; Tendulkar et al. 2017).
} 
Besides the energy scale and time scale of FRBs, another crucial constraint on models is the event rate of FRBs and, furthermore, its redshift-dependence. During the past several years, the increasing FRB number has already enabled statistical investigations of FRBs ( $\mathrm{Yu}$ et al. 2014; Bera et al. 2016; Caleb et al. 2016; Li et al. 2017; Katz 2016a; Oppermann et al. 2016; Lu \& Kumar 2016; Vedantham et al. 2016; Fialkov \& Loeb 2017; Lawrence et al. 2017; Cao et al. 2017a; Cao \& Yu 2018; Macquart \& Ekers 2018). In particular, Cao et al. (2017a) found that the proportional coefficient between the FRB rates and cosmic star formation rates (CSFRs) could be redshift-dependent, which somewhat favors the CBM model. Therefore, in this paper we confront the CBM model with the number distributions of FRBs in redshift as well as in energy (see Yamasaki et al. (2017) for a relevant calculation). By fitting the observational distributions, we test the feasibility of the CBM model for explaining the FRB phenomena and, simultaneously, constrain the model parameters. According to observational constraints, the possible nature of the progenitor compact binaries can be discussed.

\section{THE MODEL}

\subsection{The rate of $C B M s$}

A merger takes place after a compact binary loses their orbital energy through gravitational radiation. The rate of CBMs at redshift $z$ can be related to the CSFR at redshift $z^{\prime}$ that is determined by the time delay equation as $t\left(z^{\prime}\right)=t(z)-\tau$, where $t(z)=\int_{z}^{\infty}\left[\left(1+z^{\prime}\right) H\left(z^{\prime}\right)\right]^{-1} d z^{\prime}$ is the age of the universe at redshift $z$ and $H(z) \equiv$ $H_{0} \sqrt{\Omega_{\mathrm{M}}(1+z)^{3}+\Omega_{\Lambda}}$. Hereafter the cosmological parameters are taken as $\Omega_{M}=0.32, \Omega_{\Lambda}=0.68$, and $H_{0}=$ $70 \mathrm{~km} \mathrm{~s}^{-1} \mathrm{Mpc}^{-1}$. The delay time $\tau$ is determined by both the gravitational radiation decay of the binary orbit and the formation process of the compact binary. The latter factor is further related to the supernova mechanism, the natal kick velocity of NS, the mass transfer between the binary stars, and etc (Portegies Zwart \& Yungelson 1998; Belczynski et al. 2002; Mennekens et al. 2010; Chruslinska et al. 2018). Considering of a probability distribution of $P(\tau)$ of the delay times, the rate of CBMs can be calculated by the following convolution (Piran 1992; Guetta \& Piran 2006):

$$
\begin{aligned}
\dot{R}_{\mathrm{m}}(z) & \propto \int_{0}^{t(z)-t\left(z_{\mathrm{b}}\right)} \dot{\rho}_{*}[t(z)-\tau] P(\tau) d \tau \\
& \propto \int_{z}^{z_{\mathrm{b}}} \dot{\rho}_{*}\left(z^{\prime}\right) P\left[t(z)-t\left(z^{\prime}\right)\right] \frac{d t}{d z^{\prime}} d z^{\prime},
\end{aligned}
$$

where $\dot{\rho}_{*}(z)$ is the CSFR and $d t / d z=-[(1+z) H(z)]^{-1}$. The upper limit of the above integrate, $z_{\mathrm{b}}$, is set at the redshift when the binaries start forming.

Following a series of measurements of CSFRs, a consensus on the history of cosmic star formation emerges up to redshift $z \sim 4$ (Hopkins \& Beacom 2006). In the Hopkins \& Beacom's data, a trend of decrease of the CSFRs appears in higher redshift range. This trend was further confirmed by the observations of Lyman break or Ly- $\alpha$ emitter galaxies (Bouwens et al. 2012, 2015; Oesch et al. 2013, 2014; Coe et al. 2012; McLeod et al. 2016) and long GRBs (Charv et al. 2007; Yüksel et al.
2008; Kistler et al. 2009; Wang \& Dai 2009; Ishida et al. 2011; Tan et al. 2015), although there is still a debate on the decrease rate at high redshifts. The high-redshift CSFRs can also be constrained by the Gunn-Peterson trough observations to quasars and by the Thomson scattering optical depth of cosmic microwave background photons (e.g., Yu et al. 2012; Wang 2013). Combining the various measurements and constraints, we take the cosmic star formation history as follows ( $\mathrm{Yu}$ et al. 2012):

$$
\dot{\rho}_{*}(z) \propto\left\{\begin{array}{cc}
(1+z)^{3.44}, & \text { for } z<0.97, \\
(1+z)^{0}, & \text { for } 0.97 \leq z<3.5, \\
(1+z)^{-0.8}, & \text { for } z \geq 3.5,
\end{array}\right.
$$

with a local CSFR of $\dot{\rho}_{*}(0)=0.02 \mathrm{M}_{\odot} \mathrm{yr}^{-1} \mathrm{Mpc}^{-3}$. In any case, the uncertainty of the high-redshift CSFRs would not significantly influence the rates of CBMs at relatively low redshifts of most FRBs.

The lifetime of gravitational radiation decay of a binary orbit is determined by the initial orbital separation $\left(a_{i}\right)$ and the initial ellipticity $\left(e_{i}\right)$. According to the relation $\tau \propto a_{i}^{4}$ and assuming $P\left(a_{i}\right) \propto a_{i}^{q}$, Piran (1992) suggested

$$
P(\tau)=P\left(a_{i}\right) \frac{d a_{i}}{d \tau} \propto \tau^{(q-3) / 4}
$$

where the initial ellipticity is taken as a constant. A reference value of $q=-1$ can further be inferred from the data of regular binaries, which yields

$$
P(\tau) \propto 1 / \tau
$$

On the one hand, this simple power-law distribution has been generally confirmed by more elaborate calculations (Greggio 2005; Belczynski et al. 2006; Mennekens et al. 2010; Ruiter et al. 2011; Mennekens \& Vanbeveren 2016). This indicates that delay times are dominated by the gravitational radiation. On the other hand, such a delay time distribution has been widely and successfully applied in modeling the redshift distribution of SNe Ia originating from mergers of double WDs (Totani et al. 2008; Maoz \& Mannucci 2012) and of short GRBs originating from mergers of double NSs or a NS and a $\mathrm{BH}$ (Guetta \& Piran 2006; Nakar et al. 2006; Virgili et al. 2011; Hao \& Yuan 2013; Wanderman \& Piran 2015). Furthermore, this power-law distribution could also be supported by the observations of six double NS systems (Champion et al. 2004).

The delay time distribution is usually found to be peaked at a cutoff value, $\tau_{\mathrm{c}}$, below which the probability decreases drastically. Therefore, we tentatively take an empirical expression as follows:

$$
P(\tau) \propto\left(\frac{\tau}{\tau_{\mathrm{c}}}\right)^{-1} e^{-\tau_{\mathrm{c}} / \tau}
$$

with which we derive the CBM rate as a function of redshift from Eq. (11). The result is primarily dependent on the value of the crucial cutoff of the delay times, as presented in Figure 1. Numerical simulations show that the value of $\tau_{\mathrm{c}}$ is probably around a few hundred Myr for double NS mergers, but around a few ten Myr for NS-BH mergers (Mennekens \& Vanbeveren 2016; Chruslinska et al. 2018). The delay time distribution of SNe Ia can usually be described by a brokenpower law consisting of $\tau^{-0.5}$ and $\tau^{-1}$ (Greggio 2005; 
Graur \& Maoz 2013), where the former power law is probably determined by the formation time of WDs. Therefore, the value of $\tau_{\mathrm{c}}$ for double WD mergers can in principle be defined by the break time between the two power laws, which also ranges from several ten to several hundred Myr.

\subsection{Model-predicted FRB numbers}

It is assumed that a particular type of CBMs produce the observed FRBs of isotropic energy releases of $E$, which could satisfy a power-law distribution as

$$
\Phi(E) \equiv \frac{d N}{d E} \propto E^{-\gamma}, \text { for } E \geq E_{\min },
$$

where the value of $E_{\min }$ can roughly be inferred from observations. The combination of the above intrinsic energy distribution with the observational thresholds of telescopes determines the fraction of FRBs that can be detected by the telescopes. For a specific telescope survey, the observational number of FRBs in the redshift range $\left(z_{1}, z_{2}\right)$ or in the energy range $\left(E_{1}, E_{2}\right)$ can be calculated by

$N=\mathcal{T} \frac{\mathcal{A}}{4 \pi} f_{\mathrm{b}} \int_{z_{1}}^{z_{2}} \dot{R}_{\mathrm{m}}(z) \frac{d V(z)}{1+z}\left[\int_{\max \left[E_{\mathrm{th}}(z), E_{\min }\right]}^{E_{\max }} \Phi(E) d E\right]$,

or

$$
N=\mathcal{T} \frac{\mathcal{A}}{4 \pi} f_{\mathrm{b}} \int_{E_{1}}^{E_{2}} \Phi(E)\left[\int_{0}^{\min \left[z_{\mathrm{h}}(E), z_{\max }\right]} \dot{R}_{\mathrm{m}}(z) \frac{d V(z)}{1+z}\right] d E,
$$

where $\mathcal{T}$ is the duration of each pointing observation, $\mathcal{A}$ is the sky area of the survey, $f_{\mathrm{b}}$ is the beaming factor of the $\mathrm{FRB}$ radiation, $d V(z)=4 \pi d_{c}(z)^{2} c H(z)^{-1} d z$ is the comoving volume element, $d_{c}(z)=c \int_{0}^{z} H\left(z^{\prime}\right)^{-1} d z^{\prime}$ is the comoving distance, and the factor $(1+z)$ represents the cosmological time dilation for the observed rates. The energy threshold of a telescope involved in Equations (7) and (8) can be determined by

$$
E_{\mathrm{th}}(z)=4 \pi d_{c}(z)^{2}(1+z) \Delta \nu \mathcal{F}_{\nu, \mathrm{th}} k(z),
$$

where $\Delta \nu$ and $\mathcal{F}_{\nu \text { th }}$ are the frequency bandwidth and the fluence sensitivity of the telescope, respectively. The correction factor $k(z)$ converts the FRB energy from the observational band $\left(\nu_{1}, \nu_{2}\right)$ into a common emitting frequency range $\left(\nu_{a}, \nu_{b}\right)$ for all FRBs. By assuming a powerlaw spectrum, $\mathcal{F}_{\nu} \propto \nu^{-\beta}$, the $k$-correction can be calculated to

$$
k(z)=\frac{\nu_{b}^{(1-\beta)}-\nu_{a}^{(1-\beta)}}{\left[(1+z) \nu_{2}\right]^{(1-\beta)}-\left[(1+z) \nu_{1}\right]^{(1-\beta)}} .
$$

Finally, the horizon redshift $z_{\mathrm{h}}(E)$ appearing in integral (18) can be solved from the equation $E=E_{\mathrm{th}}\left(z_{\mathrm{h}}\right)$, which means that, for an isotropic energy of FRBs, the observational horizon of the telescope is at $z_{\mathrm{h}}$. The maximum redshift $z_{\max }$ corresponds to the maximum DM below which the FRB searches were conducted. One must keep in mind that a remarkable number of FRBs of relatively high redshifts and of relatively low energies have be

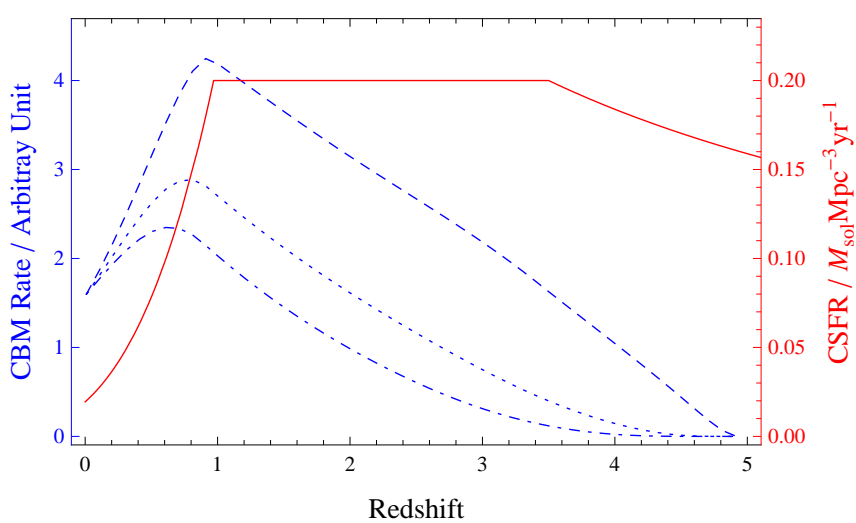

FIG. 1. - CBM rate as a function of redshift for different characteristic delay times: $\tau_{\mathrm{c}}=100,500$, and $1000 \mathrm{Myr}$ (dashed, dotted, and dash-dotted lines, respectively), where $z_{\mathrm{b}}=5$ is taken. The solid line represents the adopted star formation history.

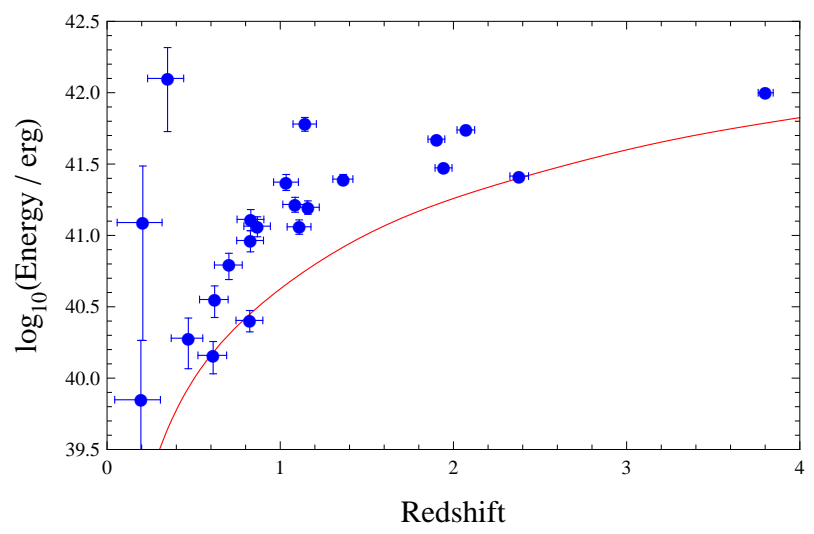

Fig. 2.- The 22 Parkes FRBs in the $z-E$ plane. The errors of the data correspond to the uncertain range of $\mathrm{DM}_{\text {host }}$ from zero to $200 \mathrm{pc} \mathrm{cm}^{-3}$ and the central values are given for $\mathrm{DM}_{\text {host }}=100 \mathrm{pc} \mathrm{cm}^{-3}$. The FRB energies are corrected for a tentative spectral index $\beta=0.8$. The solid line represents the observational energy threshold of the Parkes telescope below which the identification opportunity of an FRB decreases drastically.

missed by the present telescope surveys due to the telescope thresholds, when the observational distributions of FRBs are discussed and used.

\section{FITTING TO OBSERVATIONAL DISTRIBUTIONS}

Up to FRB 171209, a total of 30 FRBs have been detected by different telescopes including the Parkes, UTMOST, GBT, ASKAP, and Arecibo, which are cataloged on the website http://frbcat.org/ (see Petroff et al. (2016) and references therein). In this paper we only take into account the largest sub-sample provided by the Parkes containing 22 FRBs, so that the very different parameters of different telescopes will not be involved. The only repeated FRB 121102 discovered by Arecibo is just excluded. The redshifts of the FRBs can be inferred from their DMs, by subtracting the contributions from the Milky Way and the host galaxies, while the DM of the FRB sources are considered to be relatively much lower. Specifically, the following equation is used to calculate the redshifts of the FRBs (Ioka 2003; Inoue 2003; Yang \& Zhang 2017b):

$$
\operatorname{DM}_{\mathrm{IGM}}(z)=\mathrm{DM}-\mathrm{DM}_{\mathrm{MW}}-\frac{\mathrm{DM}_{\text {host }}}{1+z}
$$



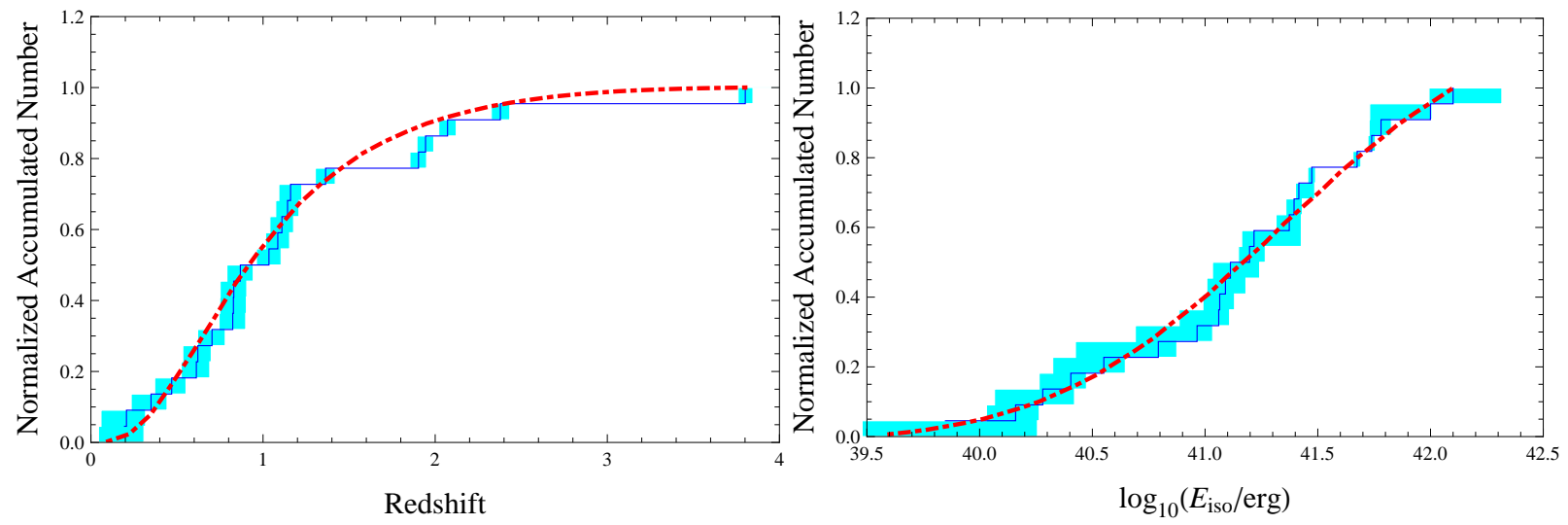

FIG. 3.- Normalized accumulated distributions in redshift (left) and isotropic energy (right) of the 22 Parkes FRBs. The solid line is obtained by fixing $\mathrm{DM}_{\text {host }}$ to $100 \mathrm{pc} \mathrm{cm}^{-3}$ for all FRBs, while the shadow represents the uncertainty of the FRB distribution arising from the variation of $\mathrm{DM}_{\text {host }}$ from $0 \mathrm{pc} \mathrm{cm}^{-3}$ to $200 \mathrm{pc} \mathrm{cm}^{-3}$. An example fitting by the CBM model to the FRB distribution for $\mathrm{DM}_{\text {host }}=100 \mathrm{pc} \mathrm{cm}^{-3}$ is presented by the dash-dotted line, where the model parameters are taken as $\beta=0.8, \gamma=1.4$, and $\tau_{\mathrm{c}}=350 \mathrm{Myr}$.
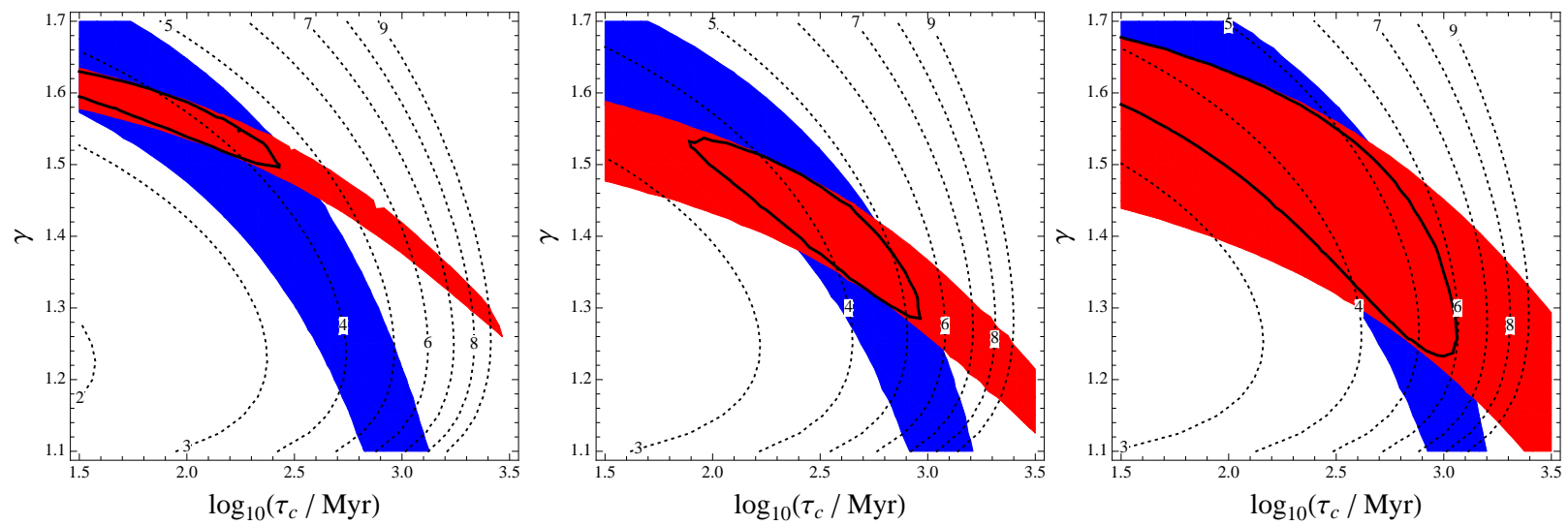

FIG. 4.- 95\% confidence level contours of parameters $\tau_{\mathrm{c}}$ and $\gamma$ given by the Kolmogorov-Smirnov test. The blue and red contours correspond to the fittings of redshift and energy distributions, respectively. The overlapped region of the contours is presented by the solid line. The dashed lines indicate the required local event rate of FRBs as labeled by $\dot{R}_{\mathrm{m}}(0) / 10^{4} \mathrm{Gpc}^{-3} \mathrm{yr}^{-1}$. From left to right, the value of $\mathrm{DM}_{\text {host }}$ is fixed to $0 \mathrm{pc} \mathrm{cm}{ }^{-3}, 100 \mathrm{pc} \mathrm{cm}^{-3}$, and $200 \mathrm{pc} \mathrm{cm}^{-3}$, respectively

$$
=f_{\mathrm{IGM}} f_{\mathrm{e}} \frac{3 c H_{0} \Omega_{\mathrm{b}}}{8 \pi G m_{\mathrm{p}}} \int_{0}^{z} \frac{H_{0}\left(1+z^{\prime}\right)}{H\left(z^{\prime}\right)} d z^{\prime},
$$

where $f_{\mathrm{IGM}} \sim 0.83$ is the fraction of baryon mass in intergalactic medium (IGM), $f_{\mathrm{e}} \sim 7 / 8$ is the number ratio between free electrons and baryons (including proton and neutron) in IGM, $m_{\mathrm{p}}$ is the proton mass, and $\Omega_{\mathrm{b}}=0.04$. While the values of $\mathrm{DM}_{\mathrm{MW}}$ have been provided in the catalog, the $\mathrm{DM}_{\text {host }}$ are completely unknown. In any case, a rough estimation on the order of magnitude of $\mathrm{DM}_{\text {host }}$ could still be made by referring to the observation of the host galaxy of FRB 121102, which gives $\mathrm{DM}_{\text {host }} \sim 100 \mathrm{pc} \mathrm{cm}^{-3}$, although it is not clear whether or not this repeated FRB has an origin identical to the non-repeated ones. Therefore, in our calculations we take the values of $\mathrm{DM}_{\text {host }}$ varying from zero

6 The DM of FRB 121102 contributed by its host galaxy was suggested by Tendulkar et al. (2017) to

$$
\mathrm{DM}_{\text {host,FRB121102 }} \approx 324 \mathrm{pc} \mathrm{cm}^{-3}\left[\frac{4 d_{\mathrm{kpc}} f}{\zeta\left(1+\epsilon^{2}\right)}\right]^{1 / 2}
$$

where $d_{\mathrm{kpc}}=d / \mathrm{kpc}$ is the total path length of the FRB emission through the galactic disk, $f$ is the faction of the path that is occupied by ionized clouds, $\zeta \geq 1$ defines cloud-to-cloud density variations in the ionized regions, $\epsilon \leq 1$ is the fractional variation inside discrete clouds due to turbulent-like density variations. to $200 \mathrm{pc} \mathrm{cm}^{-3}$, where the upper bound is set according to the present minimum DM of the Parkes FRBs, i.e., $\mathrm{DM}_{\mathrm{FRB} 150807}=266.5 \mathrm{pc} \mathrm{cm}^{-3}$. This variation range of $\mathrm{DM}_{\text {host }}$ leads to the uncertainty of redshifts and energies of the FRBs, as shown in Figure 2 .

With an inferred redshift, the isotropically equivalent energy of an FRB can be calculated by

$$
E=4 \pi d_{c}(z)^{2}(1+z) \Delta \nu \mathcal{F}_{\nu} k(z),
$$

where the Parkes parameters for $k$-correction are taken as follows: $\nu_{1}=1182 \mathrm{MHz}$ and $\nu_{2}=1522 \mathrm{MHz}$ (i.e., $\Delta \nu=0.34 \mathrm{GHz}$ centering at $1.35 \mathrm{GHz}$ ) and then $\nu_{a}=$ $1182 \mathrm{MHz}$ and $\nu_{b}=7610 \mathrm{MHz}$ which correspond to the redshift range of $0 \lesssim z \lesssim 4$ of observed FRBs. For simplicity, a tentative spectral index $\beta=0.8$ is assumed in view of our very poor knowledge of the FRBs' spectra and the high degeneracy between $\beta$ and $\gamma$ due to the $k$-correction. Two Parkes FRBs have published spectral indices including FRB 131104 with $\beta=0.3 \pm 0.9$ (Ravi et al. 2015) and FRB 150418 with $\beta=1.3 \pm 0.5$ (Keane et al. 2016). However, it should be cautioned that these values are very sensitive to the true position of the FRBs within the telescope beam pattern. Finally, the energy threshold of the Parkes telescope, presented by the solid line in Figure 2] is calculated by Equa- 
tion (9) with a fluence sensitivity as (Bera et al. 2016; Caleb et al. 2016)

$$
\mathcal{F}_{\nu, \mathrm{th}}=0.04 \frac{\mathrm{S}}{\mathrm{N}}\left[\frac{\Delta t_{\mathrm{obs}}(z)}{1 \mathrm{~ms}}\right]^{1 / 2} \mathrm{Jy} \mathrm{ms},
$$

where the characteristic minimum signal-to-noise ratio is adopted to $\mathrm{S} / \mathrm{N}=10$ and the typical FRB duration $\Delta t_{\text {obs }}(z)$ as a function of redshift is given by fitting the observational duration distribution as did in Cao et al. (2017a).

In view of the limit number of observed FRBs, we only pay attention to the accumulated distributions of the 22 Parkes FRBs, as presented in Figure 3 These FRB distributions are somewhat uncertain due to the uncertainty of $\mathrm{DM}_{\text {host }}$. For a fixed $\mathrm{DM}_{\text {host }}$, we can fit the observational distributions by Equations (7) and (8) by varying the values of the most crucial model parameters, i.e., $\tau_{\mathrm{c}}$ and $\gamma$. The goodness of the fits is assessed by the Kolmogorov-Smirnov test, where the observational uncertainties are not involved. Then, for a general consideration, we carry out such calculations for three different values of $\mathrm{DM}_{\text {host }}$, i.e., $0 \mathrm{pc} \mathrm{cm} \mathrm{cm}^{-3}, 100 \mathrm{pc} \mathrm{cm}^{-3}$, and $200 \mathrm{pc} \mathrm{cm}{ }^{-3}$. As a result, the $95 \%$ confidence level regions of parameters $\tau_{\mathrm{c}}$ and $\gamma$ are presented in Figure 4 by two contours deriving from the fittings of the redshift and energy distributions, respectively. The large overlap of the two contours demonstrates that sufficiently good fits of observations can easily be found in the CBM model. One example of the best fits to the distributions for $\mathrm{DM}_{\text {host }}=100 \mathrm{pc} \mathrm{cm}{ }^{-3}$ is showed by the dash-dotted line in Figure 3, which is given by $\tau_{\mathrm{c}}=350 \mathrm{Myr}, \gamma=1.4$, and $E_{\min }=3 \times 10^{39} \mathrm{erg}$. The results for different values of $\mathrm{DM}_{\text {host }}$ together indicate that, while $1.2 \lesssim \gamma \lesssim 1.7$, the characteristic delay time $\tau_{\mathrm{c}}$ can range from several ten to several hundred My17, which is broadly consistent with the theoretical expectations of the CBM model. This somewhat favors the CBM explanation of the FRB phenomena, although the range of $\tau_{\mathrm{c}}$ is still too large to fix the nature of the compact binaries.

Finally, by using the total FRB number of 22, we can determine the local rate of the FRB-related CBMs for different values of $\tau_{\mathrm{c}}$ and $\gamma$, as labeled by the dashed lines in Figure 4. According to the overlapped regions of the contours for all different $\mathrm{DM}_{\text {host }}$ cases, we can have

$\dot{R}_{\mathrm{m}}(0) \approx(3-6) \times 10^{4} \mathrm{Gpc}^{-3} \mathrm{yr}^{-1} f_{\mathrm{b}}^{-1}\left(\frac{\mathcal{T}}{270 \mathrm{~s}}\right)^{-1}\left(\frac{\mathcal{A}}{2 \pi}\right)^{-1}$,

where the reference values of $\mathcal{T}$ and $\mathcal{A}$ are taken by referring to Thornton et al. (2013). Substituting the above local event rate into Equation (11) and integrating $\dot{R}_{\mathrm{m}}(z)$ from $z=0$ to 4 , we can obtain the full-sky event rates for different fluence sensitivities, as listed in Table 1, where $\tau_{\mathrm{c}}=350 \mathrm{Myr}, \gamma=1.4$, and $\dot{R}_{\mathrm{m}}(0)=4.1 \times 10^{4} \mathrm{Gpc}^{-3} \mathrm{yr}^{-1}$ are taken. For the sensitivity $\mathcal{F}_{\nu \text {,th }}=0.4 \mathrm{Jy} \mathrm{ms}$ corresponding to the Parkes, the presented rate of 14,080

\footnotetext{
7 If we release the fixing of the value of $\beta$, our constraints on the model parameters can be somewhat changed, in particular, for the parameter $\gamma$ (Cao et al. 2017a) because of its tight connection with $\beta$ through the $k$-correction of FRB energies. However, the value of $\tau_{\mathrm{C}}$ would not substantially deviate from the large range presented here.
}

TABLE 1

FULL-SKy FRB EVENT RATES FOR DIFFERENT SENSITIVITIES

\begin{tabular}{|c|c|}
\hline $\begin{array}{c}\mathcal{F}_{\nu, \text { th }} \text { for } \Delta t_{\text {obs }}=1 \mathrm{~ms} \\
(\mathrm{Jy} \mathrm{ms})\end{array}$ & $\begin{array}{c}\text { Event rate } \\
\text { (Number } / \text { day } / \text { sky })\end{array}$ \\
\hline 0.2 & 20,000 \\
0.4 & 14,080 \\
1.0 & 8,100 \\
3.0 & 3,500 \\
\hline
\end{tabular}

day $^{-1} \mathrm{sky}^{-1}$ can be easily understood by the following calculation:

$$
\begin{aligned}
\dot{R}_{\mathrm{FRB}, \text { full-sky }} & =\frac{1}{f_{\mathrm{b}}} \cdot \frac{N_{\mathrm{FRB}, \text { Parkes }}}{\mathcal{T}} \cdot \frac{4 \pi}{\mathcal{A}} \\
& =14,080 \text { day }^{-1} \mathrm{sky}^{-1} f_{\mathrm{b}}^{-1}\left(\frac{\mathcal{T}}{270 \mathrm{~s}}\right)^{-1}\left(\frac{\mathcal{A}}{2 \pi}\right)^{-1}(16)
\end{aligned}
$$

\section{CONCLUSION AND DISCUSSIONS}

The fitting results presented in this paper indicate that the CBM model with reasonable parameter values can well account for the FRB phenomena in the sight of the redshift dependence of event rate, although the uncertainty of model parameters is still large. It is at least indicated that the FRB rates could be connected with CSFRs by power-law distributed delay times and the FRB energy distribution could be effectively expressed by a single power law. Furthermore, the relatively certain value of the local event rate of FRBs enables us to discuss the nature of the compact binaries, specifically, two WDs, two NSs, or a NS and a BH.

Mergers of NS-NS and NS-BH binairies are long considered to be progenitors of short GRBs, which was recently confirmed by the discovery of GRB 170817A and the associated gravitational wave event GW 170817. On the one hand, according to GW 170817, the rate of NSNS mergers has been directly inferred to $\dot{R}_{\mathrm{ns}-\mathrm{ns}}(0) \sim$ $1540_{-1220}^{+3200} \mathrm{Gpc}^{-3} \mathrm{yr}^{-1}$ (Abbott et al. 2017). An absolute upper limit on this rate was previously imposed to 12,600 $\mathrm{Gpc}^{-3} \mathrm{yr}^{-1}$ by the non-detection of this type of mergers during O1 of LIGO (Abbott et al. 2016). On the other hand, during the past decade, the local event rate of short GRBs has been widely investigated and found to be from a few to a few ten $\mathrm{Gpc}^{-3} \mathrm{yr}^{-1}$ (Guetta \& Piran 2006; Nakar et al. 2006; Guetta \& Stella 2009; Dietz 2011; Coward et al. 2012; Wanderman \& Piran 2015; Tan et al. 2018; Zhang \& Wang 2018). According to the latest statistics, we can get $\dot{R}_{\mathrm{sGRB}}(0) \approx 4 \mathrm{Gpc}^{-3} \mathrm{yr}^{-1}$ for an assumed minimum luminosity of short GRBs of $L_{\min } \sim 5 \times 10^{49} \mathrm{erg} \mathrm{s}^{-1}$. The conversion of this short GRB event rate to merger rate is highly dependent on the measurements of openning angles of GRB jets. For a possible range of the angles of $5^{\circ}-30^{\circ}$, the local merger rate can be inferred to $\dot{R}_{\mathrm{ns}-\mathrm{ns}}(0) \sim(30-1100) \mathrm{Gpc}^{-3} \mathrm{yr}^{-1}$, which is broadly in agreement with the LIGO result. Meanwhile, the rate of NS-BH mergers is considered to be comparable to or more probably lower than the rate of NS-NS mergers (Abadie et al. 2010). Therefore, it seems difficult to explain all FRBs by only NS-NS and NS-BH mergers (cf. Callister et al. 2016).

Mergers of double WDs could lead to different outcomes including SN Ia explosions, a stable WD, and 
a stable NS through accretion-induced collapse (AIC) (Canal \& Schatzman 1976; Nomoto \& Kondo 1991). Simulations showed that the local rate of WD mergers can reach several times $10^{4} \mathrm{Gpc}^{-3} \mathrm{yr}^{-1}$ (Badenes \& Maoz 2012), which was supported by the measurement of SN Ia rate as $(3.01 \pm 0.062) \times 10^{4} \mathrm{Gpc}^{-3} \mathrm{yr}^{-1}$ (Li et al. 2011) although SNe Ia can also originate from a single WD accreting from its campanion star. The general consistency between the WD merger rate and the rate presented in Equation (15), if the beaming of the FRB radiation can be ignored, indicates that the WD mergers could be the most promising origin of FRBs in the CBM scenario. So far there was no bright $\mathrm{SNe}$ Ia reported to be associated with observed FRBs. Therefore, the plausible origin of FRBs is the formation of a massive WD as suggested by Kashivama et al. (2013) or a stable AIC NS. Here, the fraction of AICs of WD-WD mergers is not clear (e.g. Yungelson \& Livio 1998). If a remarkable amount of $r$-process elements can be synthesized during AICs (Wheeler et al. 1998), the AIC rate would be constrained to be at least an order of magnitude lower than $\sim 10^{4} \mathrm{Gpc}^{-3} \mathrm{yr}^{-1}$ to be consistent with the observed abundances of neutron-rich elements in the universe (Fryer et al. 1999).

In any case, by considering of the possible high beaming of FRB radiation (i.e., $f_{\mathrm{b}} \ll 1$ ), the inferred extremely high rate of FRBs could be a serious problem for any kinds of CBMs. A possible solution of this problem is that FRBs could actually be produced by the merger products but not by the mergers themselves and, furthermore, the FRBs are all repeated just on a timescale longer than the period (i.e., several years) of current observations. If the merger products can pro- duce an FRB averagely on a timescale of $t_{v}$ during an activity period of $n t_{v}$, then the rate presented in Equation (15) can be reduced by the factor of $n$. In this case, a rapidly rotating and highly magnetized NS as a merger product could be most favorable for causing repeatable FRB radiation. This discussion is applicable for the WD AICs and also for NS-NS mergers. In the latter case, the formation of a massive NS is usually suggested by the afterglow emission of short GRBs (Dai et al. 2006; Fan \& Xu 2006; Rowlinson et al. 2013) and even by the kilonova emission (Yu \& Dai 2017). In the framework of the merger-produced NS model, the young NS could power FRBs by its rotational energy as super-giant radio pulses of pulsars (Connor et al. 2016; Cordes \& Wasserman 2016; Lvutikov 2017) or by its magnetic energy as the giant flares of Galactic magnetars (Popov \& Postnov 2010; Kulkarni et al. 2014; Katz 2016b). Additionally, a persistent counterpart associated with the FRBs can be expected to arise from the interaction of the merger/AIC ejecta with the environmental materials (Piran et al. 2013; Piro \& Kulkarni 2013). These characteristics could make regular FRBs similar to the repeated FRB 121102 (Kashivama \& Murase 2017; Metzger et al. 2017; Cao et al. 2017b; Dai et al. 2017; Michilli et al. 2018), which needs to be investigated in future.

The authors thank Bo Wang and Fa-Yin Wang for valuable discussions. This work is supported by the National Natural Science Foundation of China (grant nos. 11473008 and 11373006).

\section{REFERENCES}

Abbott, B. P., Abbott, R., Abbott, T. D., et al. 2016, Physical Review Letters, 116, 061102

Abbott, B. P., Abbott, R., Abbott, T. D., et al. 2017, Physical Review Letters, 118, 161101

Abadie, J., Abbott, B. P., Abbott, R., et al. 2010, CQGra, 27, 173001

Badenes, C. \& Maoz, D. 2012, ApJL, 749, L11

Bannister, K. W., Shannon, R. M., Macquart, J.-P., et al. 2017, ApJL, 841, L12

Bauswein, A., Goriely, S., \& Janka, H.-T. 2013, ApJ, 773, 78

Belczynski, K., Kalogera, V., \& Bulik, T. 2002, ApJ, 572, 407

Belczynski, K., Perna, R., Bulik, T., et al. 2006, ApJ, 648, 1110

Bera, A., Bhattacharyya, S., Bharadwaj, S., Bhat, N. D. R., \& Chengalur, J. N. 2016, MNRAS, 457, 2530

Bouwens, R. J., Illingworth, G. D., Oesch, P. A., et al. 2012, ApJ, 754, 83

Bouwens, R. J., Illingworth, G. D., Oesch, P. A., et al. 2015, ApJ, 803,34

Burke-Spolaor, S., \& Bannister, K. W. 2014, ApJ, 792, 19

Callister, T., Kanner, J., \& Weinstein, A. 2016, ApJL, 825, L12

Caleb, M., Flynn, C., Bailes, M., et al. 2016, MNRAS, 458, 708

Caleb, M., Flynn, C., Bailes, M., et al. 2017, MNRAS, 468, 3746

Canal, R., \& Schatzman, E. 1976, A\&A, 46, 229

Cao, X.-F., Xiao, M., \& Xiao, F. 2017a, Research in Astronomy and Astrophysics, 17, 14

Cao, X.-F., Yu, Y.-W., \& Dai, Z.-G. 2017b, ApJ, 839, L20

Cao, X.-F., \& Yu, Y.-W. 2018, Phys. Rev. D, 97, 023022

Champion, D. J., Lorimer, D. R., McLaughlin, M. A., et al. 2004, MNRAS, 350, L61

Champion, D. J., Petroff, E., Kramer, M., et al. 2016, MNRAS, 460, L30

Chary, R., Berger, E., \& Cowie, L. 2007, ApJ, 671, 272

Chatterjee, S., Law, C. J., Wharton, R. S., et al. 2017, Nautre, 541,58
Chruslinska, M., Belczynski, K., Klencki, J., \& Benacquista, M. 2018, MNRAS, 474, 2937

Coe, D., Umetsu, K., Zitrin, A., et al. 2012, ApJ, 757, 22

Connor, L., Sievers, J., \& Pen, U.-L. 2016, MNRAS, 458, L19

Cordes, J. M., \& Wasserman, I. 2016, MNRAS, 457, 232

Coulter, D. A., Foley, R. J., Kilpatrick, C. D., et al., 2017, Science, 358, 1556

Coward, D. M., Howell, E. J., Piran, T., et al. 2012, MNRAS, 425,2668

Dai, Z. G., Wang, X. Y., Wu, X. F., \& Zhang, B. 2006, Science, 311,1127

Dai, Z. G., Wang, J. S., \& Yu, Y. W. 2017, ApJL, 838, L7

Dietz, A. 2011, A\&A, 529, A97

Eichler, D., Livio, M., Piran, T., \& Schramm, D. N. 1989, Nature, 340,126

Evans, P. A., Cenko, S. B., Kennea, J. A., et al., 2017, Science, 358,1565

Fan, Y.-Z., \& Xu, D. 2006, MNRAS, 372, L19

Fialkov, A., \& Loeb, A, 2017, ApJL, 846, L27

Fryer, R. J., Benz, W., Herant, M., \& Colgate, S. A. 1999, ApJ, 516,892

Graur, O., \& Maoz, D. 2013, MNRAS, 430, 1746

Greggio, L. 2005, A\&A, 441, 1055

Guetta, D., \& Piran, T. 2006, A\&A, 453, 823

Guetta, D., \& Stella, L. 2009, A\&A, 498, 329

Hao, J.-M., \& Yuan, Y.-F. 2013, A\&A, 558, A22

Hopkins, A. M., \& Beacom, J. F. 2006, ApJ, 651, 142

Hotokezaka, K., Kiuchi, K., Kyutoku, K., et al. 2013,

Phys. Rev. D, 87, 024001

Inoue, A. K. 2003, PASJ, 55, 901

Ishida, E. E. O., de Souza, R. S., \& Ferrara, A. 2011, MNRAS, 418,500

Ioka, K. 2003, ApJL, 598, L79 
Just, O., Bauswein, A., Pulpillo, R. A., Goriely, S., \& Janka, H.-T. 2015, MNRAS, 448, 541

Kashiyama, K., Ioka, K., \& Mészáros, P. 2013, ApJ, 776, L39

Kashiyama, K., \& Murase, K. 2017, ApJ, 839, L3

Katz, J. I. 2016a, ApJ, 818, 19

Katz, J. I. 2016b, ApJ, 826, 226

Keane, E. F., Johnston, S., Bhandari, S., et al. 2016, Nature, 530, 453

Keane, E. F., Stappers, B. W., Kramer, M., \& Lyne, A. G. 2012, MNRAS, 425, L71

Kistler, M. D., Yüksel, H., Beacom, J. F., Hopkins, A. M., \& Wyithe, J. S. B. 2009, ApJ, 705, L104

Kulkarni, S. R., Ofek, E. O., Neill, J. D., Zheng, Z., \& Juric, M. 2014, ApJ, 797, 70

Lattimer, J. M., \& Schramm, D. N. 1974, ApJ, 192, L145

Lawrence, E., Vander Wiel, S., Law, C., et al. 2017, AJ, 154, 117

Li, L.-X., \& Paczyński, B. 1998, ApJ, 507, L59

Li, L.-B., Huang, Y.-F., Zhang, Z.-B., Li, D., \& Li, B. 2017, Research in Astronomy and Astrophysics, 17, 6

Li, W., Chornock, R., Leaman, J., et al. 2011, MNRAS, 412, 1473

Lorimer, D. R., Bailes, M., McLaughlin, M. A., Narkevic, D. J., \& Crawford, F. 2007, Science, 318, 777

Lu, W. B., \& Kumar, P. 2016, MNRAS, 461, L122

Lyutikov, M. 2017, ApJ, 838, L13

Macquart, J.-P., \& Ekers, R. D. 2018, MNRAS, 474, 1900

Masui, K., Lin, H.-H., Sievers, J., et al. 2015, Nature, 528, 523

Maoz, D., \& Mannucci, F. 2012, PASA, 29, 447

Marcote, B., Paragi, Z., Hessels, J. W. T., et al. 2017, ApJ, 834, L8

McLeod, D. J., McLure, R. J., \& Dunlop, J. S. 2016, MNRAS, 459,3812

Mennekens, N., \& Vanbeveren, D. 2016, A\&A, 589, A64

Mennekens, N., Vanbeveren, D., De Greve, J. P., \& De Donder, E. 2010, A\&A, 515, A89

Metzger, B. D., Martínez-Pinedo, G., Darbha, S., et al. 2010, MNRAS, 406, 2650

Metzger, B. D., Berger, E., \& Margalit, B. 2017, ApJ, 841, 14

Michilli, D., Seymour, A., Hessels, J. W. T., et al. 2018, Nature, 553,182

Mingarelli, C. M. F., Levin, J., \& Lazio, T. J. W. 2015, ApJ, 814, L20

Nakar, E., Gal-Yam, A., \& Fox, D. B. 2006, ApJ, 650, 281

Nomoto, K., Iwamoto, K., Nakasato, N., et al. 1997, Nuclear Physics A, 621, 467

Nomoto, K., \& Kondo, Y. 1991, ApJ, 367, L19

Oesch, P. A., Bouwens, R. J., Illingworth, G. D., et al. 2013, ApJ 773,75

Oesch, P. A., Bouwens, R. J., Illingworth, G. D., et al. 2014, ApJ, 786,108

Oppermann, N., Connor, L. D., \& Pen, U.-L. 2016, MNRAS, 461, 984

Paczynski, B. 1986, ApJ, 308, L43

Petroff, E., Barr, E. D., Jameson, A., et al. 2016, PASA, 33, e045

Petroff, E., Burke-Spolaor, S., Keane, E. F., et al. 2017, MNRAS, 469,4465
Piran, T. 1992, American Institute of Physics Conference Series, 272, 1626

Piran, T., Nakar, E., \& Rosswog, S. 2013, MNRAS, 430, 2121

Piro, A. L. \& Kulkarni, S. R. 2013, ApJL, 762, L17

Popov, S. B., \& Postnov, K. A. 2010, Evolution of Cosmic Objects through their Physical Activity, 129

Portegies Zwart, S. F., \& Yungelson, L. R. 1998, A\&A, 332, 173

Ravi, V., Shannon, R. M., \& Jameson, A. 2015, ApJ, 799, L5

Rowlinson, A., O'Brien, P. T., Metzger, B. D., Tanvir, N. R., \& Levan, A. J. 2013, MNRAS, 430, 1061

Ruiter, A. J., Belczynski, K., Sim, S. A., et al. 2011, MNRAS, 417, 408

Spitler, L. G., Cordes, J. M., Hessels, J. W. T., et al. 2014, ApJ, 790, 101

Tan, W.-W., Cao, X.-F., \& Yu, Y.-W. 2015, New Astron., 38, 11

Tan, W.-W., Fan, X.-L., Wang, F. Y. 2018, MNRAS, 475, 1331

Tendulkar, S. P., Bassa, C. G., Cordes, J. M., et al. 2017, ApJ, 834, L7

Thornton, D., Stappers, B., Bailes, M., et al. 2013, Science, 341, 53

Totani, T. 2013, PASJ, 65, L12

Totani, T., Morokuma, T., Oda, T., Doi, M., \& Yasuda, N. 2008, PASJ, 60, 1327

Tutukov, A. V., \& Yungelson, L. R. 1981, Nauchnye Informatsii, 49,3

Vedantham, H. K., Ravi, V., Hallinan, G., et al. 2016, ApJ, 830, 75

Virgili, F. J., Zhang, B., Nagamine, K., \& Choi, J.-H. 2011, MNRAS, 417, 3025

Wanderman, D., \& Piran, T. 2015, MNRAS, 448, 3026

Wang, B., \& Han, Z. 2012, New Astro. Rev., 56, 122

Wang, F. Y., \& Dai, Z. G. 2009, MNRAS, 400, L10

Wang, F. Y. 2013, A\&A, 556, A90

Wang, J.-S., Yang, Y.-P., Wu, X.-F., Dai, Z.-G., \& Wang, F.-Y. 2016, ApJ, 822, L7

Webbink, R. F. 1984, ApJ, 277, 355

Wheeler, J. C., Cowan, J. J., Hillebrandt, W. 1998, ApJ, 493, L101

Yang, Y. P. \& Zhang, B. 2017a, arXiv: 1712.02702

Yang, Y. P. \& Zhang, B. 2017b, 2017, ApJ, 847, 22

Yüksel, H., Kistler, M. D., Beacom, J. F., \& Hopkins, A. M. 2008, ApJ, 683, L5

Yamasaki, S., Totani, T., \& Kiuchi, K. 2017, arXiv:1710.02302

Yu, Y.-W., \& Dai, Z.-G. 2017, arXiv:1710.01898

Yu, Y.-W., Cheng, K. S., Chu, M. C., \& Yeung, S. 2012, JCAP, 7, 023

Yu, Y.-W., Cheng, K.-S., Shiu, G., \& Tye, H. 2014, JCAP, 11, 040

Yu, Y.-W., Zhang, B., \& Gao, H. 2013, ApJ, 776, L40

Yungelson, L., \& Livio, M. 1998, ApJ, 497, 168

Zhang, B. 2016, ApJ, 827, L31

Zhang, G. Q., \& Wang, F. Y. 2018, ApJ, 852, 1 